\title{
Modification of surgical procedure for "probable" limited dorsal myeloschisis
}

\author{
Ji Yeoun Lee, MD, PhD, ${ }^{1,2}$ Sangjoon Chong, MD, ${ }^{1}$ Young Hun Choi, MD, ${ }^{3}$ Ji Hoon Phi, MD, PhD, ${ }^{1}$ \\ Jung-Eun Cheon, MD, PhD, ${ }^{3}$ Seung-Ki Kim, MD, PhD, ${ }^{1}$ Sung Hye Park, MD, PhD, ${ }^{4}$ \\ In-One Kim, MD, PhD, ${ }^{3}$ and Kyu-Chang Wang, MD, PhD ${ }^{1}$
}

\begin{abstract}
Divisions of ${ }^{1}$ Pediatric Neurosurgery and ${ }^{3}$ Pediatric Radiology, Seoul National University Children's Hospital; ${ }^{4}$ Department of Pathology, Seoul National University Hospital; and 'Department of Anatomy, Seoul National University College of Medicine, Seoul, Korea
\end{abstract}

\begin{abstract}
OBJECTIVE Since the entity limited dorsal myeloschisis (LDM) was proposed, numerous confusing clinical cases have been renamed according to the embryopathogenesis. However, clinical application of this label appears to require some clarification with regard to pathology. There have been cases in which all criteria for the diagnosis of LDM were met except for the presence of a neural component in the stalk, an entity the authors call "probable" LDM. The present study was performed to meticulously review these cases and suggest that a modified surgical strategy using limited laminectomy is sufficient to achieve the surgical goal of untethering.

METHODS The authors retrospectively reviewed the imaging findings, operative notes, and pathology reports of spinal dysraphism patients with subcutaneous stalk lesions who had presented to their institution between 2010 and 2014.

RESULTS Among 33 patients with LDM, 13 had the typical nonsaccular lesions with simple subcutaneous stalks connecting the skin opening to the spinal cord. Four cases had "true" LDM meeting all criteria for diagnosis, including pathological confirmation of CNS tissue by immunohistochemical staining with glial fibrillary acidic protein. There were also 9 cases in which all clinical, imaging, and surgical findings were compatible with LDM, but the "neural" component in the resected stalk was not confirmed. For all the cases, limited exposure of the stalk was done and satisfactory untethering was achieved.
\end{abstract}

CONCLUSIONS One can speculate based on the initial error of embryogenesis that if the entire stalk were traced to the point of insertion on the cord, the neural component would be proven. However, this would require an extended level of laminectomy/laminotomy, which may be unnecessary, at least with regard to the completeness of untethering. Therefore, the authors propose that for some selected cases of LDM, a minimal extent of laminectomy may suffice for untethering, although it may be insufficient for diagnosing a true LDM.

https://thejns.org/doi/abs/10.3171/2016.12.PEDS16171

KEY WORDS limited dorsal myeloschisis; modified surgical procedure; laminectomy; spine

$\mathrm{T}$ HE diverse and confusing morphology of spinal dysraphism halts prompt, accurate diagnosis and has resulted in numerous misnomers. It appears that disease classification based on the common error in embryogenesis is essential for correct diagnosis and treatment, especially as regards surgical strategy. One of the most recently compiled disease entities is limited dorsal myeloschisis (LDM), which is characterized by "a focal 'closed' midline defect and a fibroneural stalk that links the skin lesion to the underlying cord." ${ }^{\prime, 5}$ These 2 features reflect the fundamental error during embryogenesis that causes LDM: incomplete disjunction between the skin and neural ectoderm during primary neurulation.

Focusing on the underlying embryogenesis and excluding the prejudices of gross morphology and location, several controversial entities have been classified as LDM, such as cervical myelomeningocele, spinal meningocele, and "skin covered myelomeningocele.", $, 7,8$ We have also been more alert in the diagnosis and treatment of LDM. However, we have encountered cases in which all criteria for the diagnosis of LDM were met except for the presence of a neural component in the stalk. Based on the embryol-

ABBREVIATIONS CNS = central nervous system; EMG = electromyography; GFAP = glial fibrillary acidic protein; IHC = immunohistochemistry; LDM = limited dorsal myeloschisis; UDS = urodynamic study.

SUBMITTED March 17, 2016. ACCEPTED December 2, 2016.

INCLUDE WHEN CITING Published online February 17, 2017; DOI: 10.3171/2016.12.PEDS16171. 
ogy, it can be speculated that the neural component would have been found by exploration of the entire stalk; hence, we call these lesions "probable" LDMs. The present study was performed to meticulously review these cases and suggest that a modified surgical strategy using limited laminectomy for these probable LDMs is sufficient to achieve the surgical goal of untethering.

\section{Methods}

The recorded operation database was searched, and all patients who had undergone surgery for congenital spinal dysraphism between 2010 and 2014 were identified. All patients had undergone preoperative lumbosacral MRI. Preoperative urodynamic study (UDS) and electromyography (EMG) were usually recommended and performed, but some of the asymptomatic, young patients were spared from undergoing these additional tests. Intraoperative electrophysiological monitoring was performed in all patients. Disease entities included spinal lipomas, Currarino triad, congenital dermal sinus, myelomeningocele, terminal myelocystocele, thick and short filum, and LDM. Among the 227 identified patients, there were 33 with diagnosed LDM, including the saccular and nonsaccular types. The radiological studies, operative findings, and histopathological results of these LDM patients were thoroughly reviewed. For the purposes of this study, LDM cases with associated lesions such as lipoma, congenital dermal sinus, or dermoid cyst were excluded, and only nonsaccular-type lesions were considered. The Institutional Review Board of Seoul National University Hospital approved the study.

The criteria for probable LDM were as follows: 1) a typical "flat" skin lesion (for example, midline crater or pit or cigarette-burn scar); 2) a stalk connecting the base of the skin lesion to a "limited" area of the spinal cord, as seen on MRI; and 3) a dural fistula ensheathing the connecting stalk. The lesions would otherwise be a very typical nonsaccular type of LDM, except that central nervous system (CNS) tissue was not found in the connecting stalk in the available surgical specimen.

The expression of glial fibrillary acidic protein (GFAP) was evaluated for the presence of CNS tissue using immunohistochemistry (IHC). Formalin-fixed, paraffin-embedded tissue blocks were used, and IHC was performed using polyclonal GFAP antibody (1:300 dilution, Dako) with the BenchMark XT automated system (Ventana Medical Systems). The positive control was normal human brain including gray and white matter, and for the negative control in IHC, the blood vessels in the samples were used. A neuropathologist (S.H.P.) evaluated the results under a microscope. Staining was read as positive or negative. Positive staining showed homogenous intensity and robust positivity; therefore, intensity and percentage of positive area were not graded.

\section{Results}

Of the 33 patients with LDM, 13 had the typical nonsaccular lesions with simple subcutaneous stalks connecting the skin lesion to the spinal cord. Four patients had "true" LDMs meeting all criteria for diagnosis, including pathological confirmation of CNS tissue by immunohistochemical staining with GFAP. However, the surgically resected stalks of the 9 remaining patients did not contain CNS tissue, meeting the criteria for probable LDM. The remaining 20 patients were excluded from the study because they harbored saccular-type lesions or had associated lesions, such as lipomas.

\section{Clinical Details}

Nine patients met the definition of probable LDM cases. The median age was 4 months at operation (range 2-72 months), and 7 patients were girls. All patients had a presenting symptom of "skin lesion," and all were neurologically asymptomatic. Skin lesions consisted of a cigaretteburn lesion (6 cases), small pit (2 cases), or skin tag (1 case). Hemangioma was observed in 6 patients. Magnetic resonance imaging revealed a typical slender stalk connecting the skin lesion to the spinal cord, and the extraspinal and intradural course could be traced as expected for an LDM (Fig. 1). The results of preoperative UDS and EMG were all normal. Thus, the patients underwent surgery with the suspected preoperative diagnosis of LDM. After the operations, all patients remained symptom free during a mean follow-up of 3.0 years (range 1.8-5.5 years; Table 1).

\section{Surgical Procedures and Pathological Findings}

The stalk was traced to a lamina defect, and limited opening of the lamina was performed. Two-level laminotomy and laminoplasty was performed in 5 patients, and 1to 1.5-level hemilaminectomy was performed in 4 patients (Fig. 2). Laminotomy was preferred in the early period, but hemilaminectomy has become the preferred method of removing laminae. A slender dural fistula wrapping the stalk was identified in all cases, and after dural opening, the stalk was sectioned, completing the untethering. Frozen biopsy procedure was performed to check for cutaneous ectoderm in the proximal stalk. The filum was cut based on surgeon preference (7 cases). The site of stalk attachment to the spinal cord was not visible in the surgical field in any of the cases. Pathological examination of the resected stalk confirmed the presence of either fibroadipose or fibrovascular tissue, and some samples also contained peripheral nerve twigs (5 cases). All of these stalks were negative for GFAP.

\section{Discussion}

We have shared our experience with patients whose lesions almost fit the definition of a nonsaccular LDM except for the pathological confirmation of CNS tissue in the stalk. As neural tissue may be present in the more proximal portion of the stalk, exposure and resection of the entire length of the stalk may be necessary to pathologically confirm the neural component. However, in our cases, the goal of lamina opening was visualization of the intradural portion of the stalk, which is sufficient for complete untethering. Therefore, a segment of the proximal stalk was not resected and could not be examined for pathology. Nonetheless, despite the uncertain diagnoses, complete untethering was achieved in all cases, and the surgical 

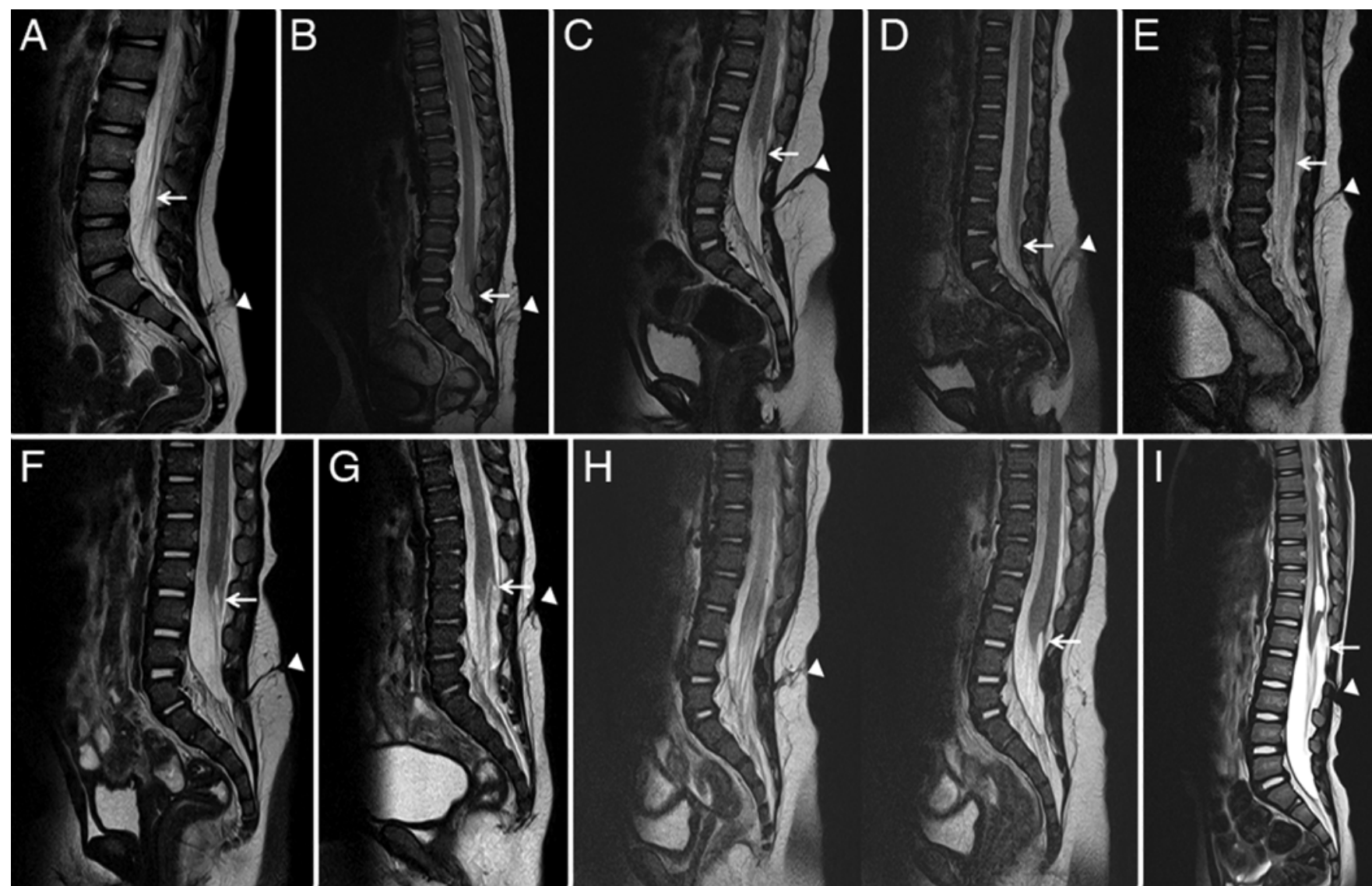

FIG. 1. Sagittal MR images obtained in 9 patients, showing the subcutaneous (arrowheads) and intradural (arrows) tracts typical of an LDM.

outcomes were favorable with considerably smaller laminectomies. We propose that for patients with the typical skin lesion and MRI features of probable LDM, the extent of laminectomy can be minimized even though the pathological diagnosis may not be confirmed.

The importance of classifying congenital spinal dysraphism according to the embryological mechanism cannot be overemphasized. An understanding of the embryopathogenesis recently led to the notion that a dysraphic spinal lipoma can be "separated" from the spinal cord. From this concept, a completely different surgical strategy of total resection for congenital spinal lipomas was derived and has greatly improved surgical outcomes. ${ }^{3}$ Similarly, before a complete understanding of the embryological origin and morphology of LDM, saccular lesions in the cervical region had been erroneously treated with ligation and cosmetic restoration of the large sac. ${ }^{2}$ It is now clear that the primary goal of surgically treating LDM is the elimination of the tethering on the cord caused by the fibroneural stalk. In the original report, Pang and colleagues noted that at least 1 set of laminae rostral and caudal to the cord-stalk attachment site usually had to be removed for sufficient visualization of the dural outpouching and the interface between cord and stalk. ${ }^{4,5}$ The entire length of the stalk was removed and available for pathological evaluation. In our experience, however, the primary tethering point appears to be on the skin and at the site of dural entry. Therefore, exposure of the entire extent of the intradural stalk was not essential for the untethering procedure. Cutting the intradural stalk and removing the distal stalk result in efficient untethering. The main advantage of this alternate procedure is the smaller extent of laminectomy. In almost half of the patients in this study, the untethering and filum sectioning were performed without difficulty after hemilaminectomy of 1-2 laminae, whereas the origi-

TABLE 1. Summary of clinical data in 9 patients with probable LDM

\begin{tabular}{|c|c|c|c|c|c|c|}
\hline $\begin{array}{l}\text { Case } \\
\text { No. }\end{array}$ & $\begin{array}{c}\text { Age at Op } \\
\text { (mos) }\end{array}$ & $\begin{array}{l}\text { Preop } \\
\text { Motor }\end{array}$ & $\begin{array}{l}\text { Preop } \\
\text { Voiding }\end{array}$ & $\begin{array}{c}\text { FU } \\
\text { (yrs) }\end{array}$ & $\begin{array}{l}\text { Motor at } \\
\text { Last FU }\end{array}$ & $\begin{array}{l}\text { Voiding at } \\
\text { Last FU }\end{array}$ \\
\hline 1 & 4 & $W N L^{*}$ & $W_{N L^{*}}$ & 5.5 & $W_{N L^{*}}$ & WNL \\
\hline 2 & 2 & $W_{N L^{*}}$ & $W_{N L}^{*}$ & 3.7 & $W_{N L}^{*}$ & WNL \\
\hline 3 & 5 & $W_{N L^{*}}$ & $W_{N L^{*}}$ & 3 & WNL & WNL \\
\hline 4 & 4 & $W N L^{*}$ & $W_{N L^{*}}$ & 3 & WNL & WNL \\
\hline 5 & 36 & $W_{N L^{*}}$ & WNL & 3 & $W_{N L^{*}}$ & WNL \\
\hline 6 & 4 & $W_{N L^{*}}$ & $W_{N L^{*}}$ & 3.2 & $W_{N L^{*}}$ & WNL \\
\hline 7 & 3 & $W_{N L^{*}}$ & $W_{N L^{*}}$ & 2.6 & WNL & WNL \\
\hline 8 & 4 & $W_{N L^{*}}$ & $W_{N L^{*}}$ & 1.8 & WNL & WNL \\
\hline 9 & 72 & $W_{N L^{*}}$ & $W_{N L^{*}}$ & 2 & WNL & WNL \\
\hline
\end{tabular}

$\mathrm{FU}=$ follow-up; $\mathrm{WNL}=$ within normal limits.

* Confirmed by EMG or UDS, as appropriate. 


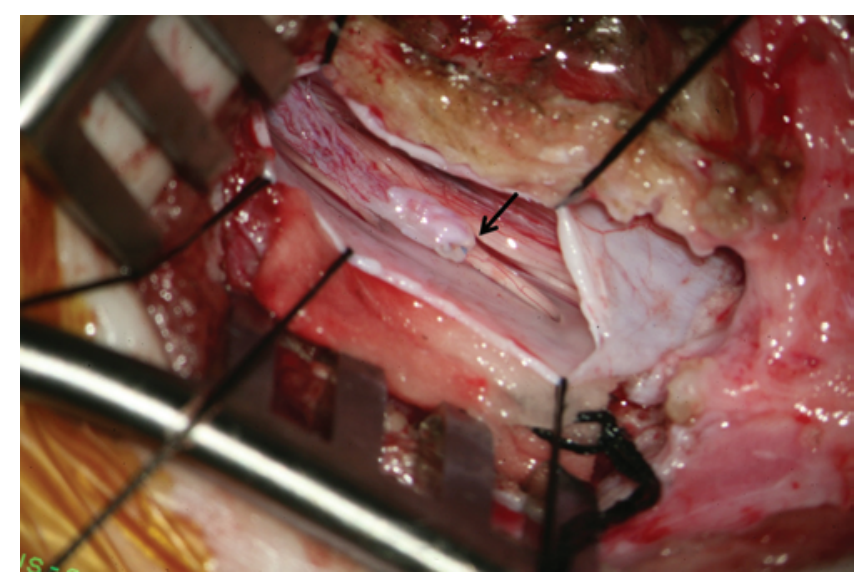

FIG. 2. Microscopic view of intradural space after hemilaminectomy of a portion of L-2 and L-3. Arrow indicates the cut proximal end of the tract. Figure is available in color online only.

nal procedure necessitates the removal of at least 2 sets of laminae. The major pitfall of the limited exposure is the possibility of not detecting the neural component and thus affecting the diagnosis of LDM in a strict sense. Although laminectomy of the lumbar level during childhood can be tolerated, it is not without risk. ${ }^{6}$ We believe this should be avoided when possible, even at the cost of a "probable" diagnosis.

One caveat of the limited approach should be noted. Only a small number of patients were evaluated, and although the duration of follow-up was fairly long, since retethering can occur even years after operation, long-term follow-up with more patients is needed.

\section{Conclusions}

We suggest that for patients with typical nonsaccular LDM-like clinical features and a simple stalk, untethering of the stalk can be achieved through limited intradural access without exposing the stalk-cord attachment site. This approach may spare the patient from multiple-level laminectomies but may halt the pathological confirmation of neural tissue in the stalk. Long-term follow-up with a greater number of patients is needed to check on the completeness of untethering and the possibility of the formation of dermoid tumors from residual cutaneous components.

\section{Acknowledgments}

This work was supported by the Research Resettlement Fund for the new faculty of Seoul National University. This research was supported by the Basic Science Research Program through the National Research Foundation of Korea (NRF) funded by the Ministry of Education (2015R1D1A1A01059605).

\section{References}

1. Habibi Z, Nejat F, Tajik P, Kazmi SS, Kajbafzadeh AM: Cervical myelomeningocele. Neurosurgery 58:1168-1175, 2006

2. Meyer-Heim AD, Klein A, Boltshauser E: Cervical myelomeningocele-follow-up of five patients. Eur J Paediatr Neurol 7:407-412, 2003

3. Pang D, Zovickian J, Oviedo A: Long-term outcome of total and near-total resection of spinal cord lipomas and radical reconstruction of the neural placode: part I-surgical technique. Neurosurgery 65:511-529, 2009

4. Pang D, Zovickian J, Oviedo A, Moes GS: Limited dorsal myeloschisis: a distinctive clinicopathological entity. Neurosurgery 67:1555-1580, 2010

5. Pang D, Zovickian J, Wong ST, Hou YJ, Moes GS: Limited dorsal myeloschisis: a not-so-rare form of primary neurulation defect. Childs Nerv Syst 29:1459-1484, 2013

6. Papagelopoulos PJ, Peterson HA, Ebersold MJ, Emmanuel PR, Choudhury SN, Quast LM: Spinal column deformity and instability after lumbar or thoracolumbar laminectomy for intraspinal tumors in children and young adults. Spine (Phila Pa 1976) 22:442-451, 1997

7. Rossi A, Piatelli G, Gandolfo C, Pavanello M, Hoffmann C, Van Goethem JW, et al: Spectrum of nonterminal myelocystoceles. Neurosurgery 58:509-515, 2006

8. Salomão JF, Cavalheiro S, Matushita H, Leibinger RD, Bellas AR, Vanazzi E, et al: Cystic spinal dysraphism of the cervical and upper thoracic region. Childs Nerv Syst 22:234-242, 2006

\section{Disclosures}

The authors report no conflict of interest concerning the materials or methods used in this study or the findings specified in this paper.

\section{Author Contributions}

Conception and design: Wang, Lee. Acquisition of data: Lee, Chong, Choi, Cheon, Park. Analysis and interpretation of data: Lee, Choi, Cheon, Park, IO Kim. Drafting the article: Wang, Lee, SK Kim. Critically revising the article: Wang, Lee, Phi, Cheon, SK Kim, IO Kim. Reviewed submitted version of manuscript: Wang, Lee, Choi, Phi, Cheon, SK Kim, Park, IO Kim. Approved the final version of the manuscript on behalf of all authors: Wang. Statistical analysis: Lee. Administrative/technical/material support: Chong, SK Kim. Study supervision: Wang.

\section{Correspondence}

Kyu-Chang Wang, Division of Pediatric Neurosurgery, Seoul National University Children's Hospital, 101 Daehak-ro, Jongnogu, Seoul 110-744, Republic of Korea. email: kcwang@snu.ac.kr. 